\title{
Seizing control over quantum systems through measurement
}
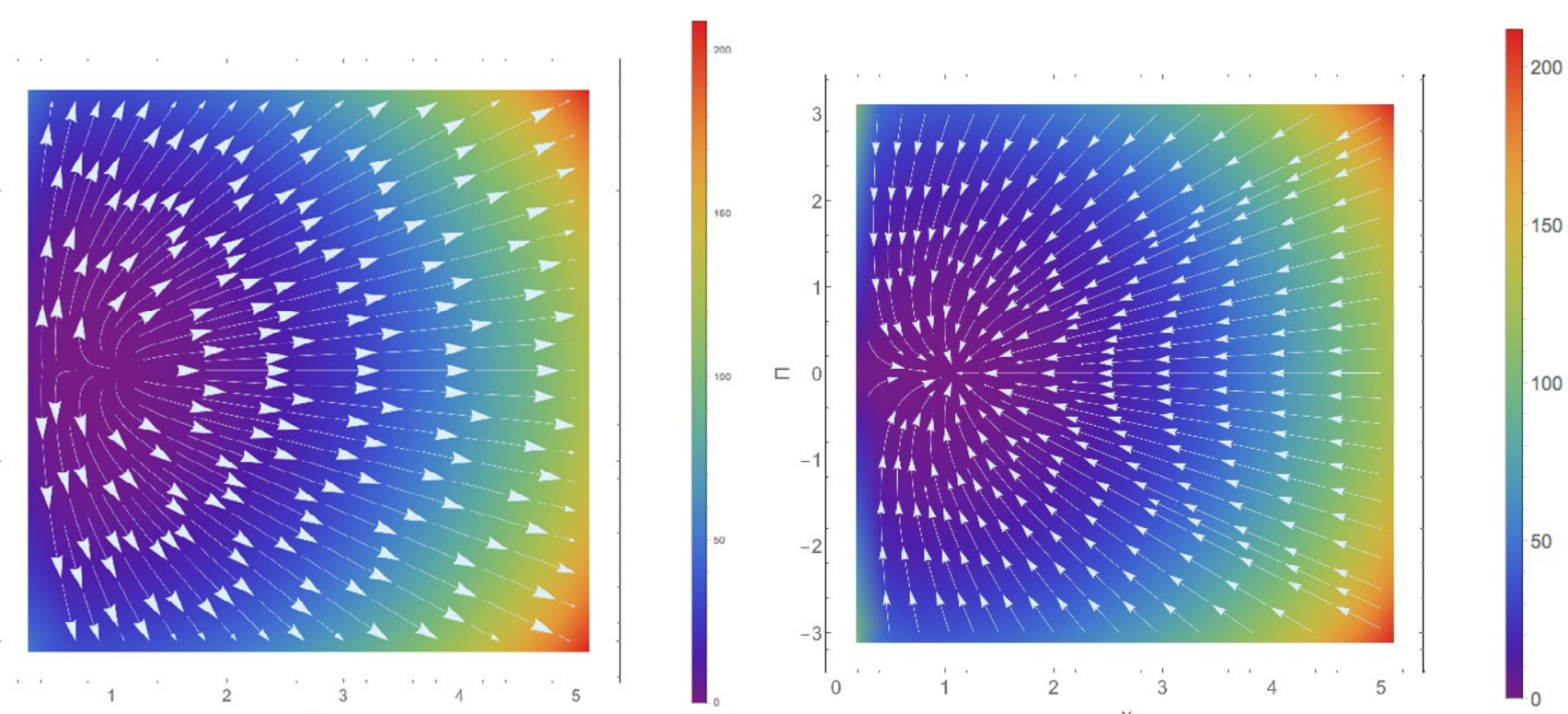

Thanks to the deep complexities of quantum mechanics, physicists have so far faced serious difficulties over quantum systems. Pattanayak at Carleton College in Minnesota demonstrates that the dynamics of used to measure them. His team's results could lead to a breakthrough in the capabilities of quantum experiments, but may also our understanding of the flow his research, Dr Arjendu vibrating quantum systems can be altered by adjusting have profound implications for

erhaps one of the most well-known
properties of quantum systems is that they can exist in multiple on until the mont they are 'observed'. When their properties are measured these clouds of possible states 'collapse' into just one, which we perceive as the sole outcome of the observation. Because of this unavoidable property, physicists over the past several decades have often viewed quantum systems as being too complex and unpredictable to establish any reasonable degree of control over. Wit recent research, however, this picture has been changing quickly.

"Quantum systems evolve as states that are in a 'superposition' over many potential results of measurements," Dr Pattanayak explains. "Interacting considerably but now dass, we

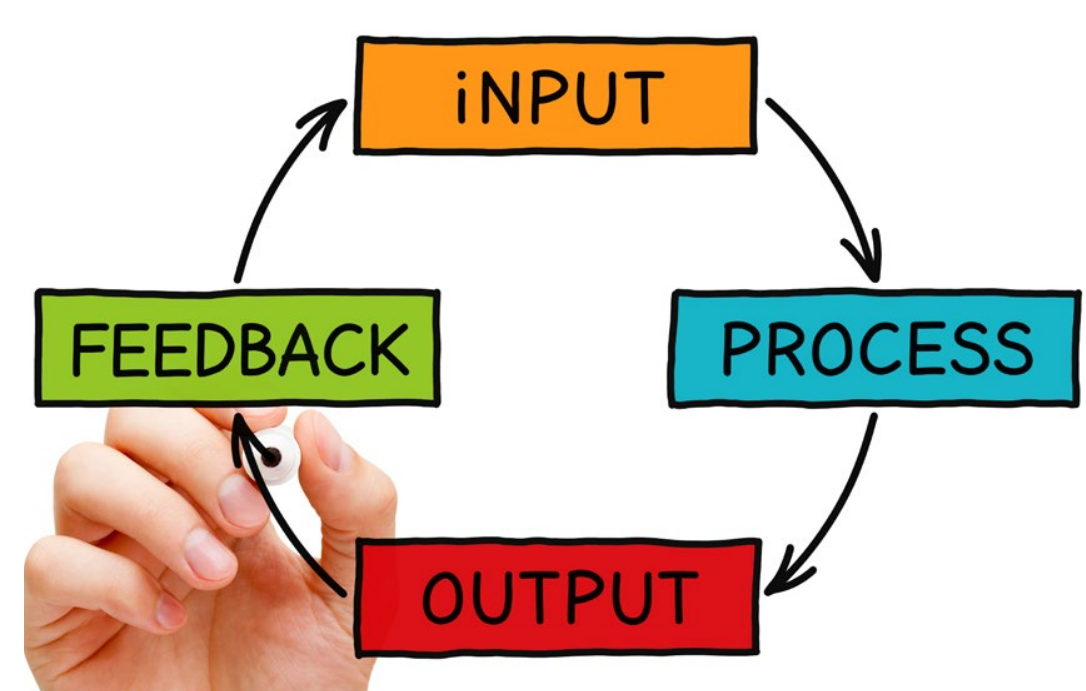

are learning how to exploit this measurement "back action' in unusual and potentially valuable ways." (c) 'quantum nonlinear oscillators' - system which vibrate in ways which don't intuitively reflect the forces acting upon them, and gradually dissipate energy into their surroundings over time.

In his work, Dr Pattanayak explores how the very devices used to measure these systems can be set to alter the ways in which energy flows through them, varying their dynamics in turn.

REDUCED, BUT NOT SINGLE STATES Before they are observed in real experiments, many physical ideas are theories As Dr Pattangat mathematical physicists how experimental obsenvitions made with present-day technology may reduce the number of states a system could possibly be in, but not down to a single

Until recently, quantum measurements were associated with a 'projective collapse' of the range of potential values to one specific sharp result," he says. "We now use more "bluryy" ceasurements which cause smaller collapse - a relatively far smaller reduction in the range of potential values compared to the original state. nevitably, the theories required to describe how a cloud of quired to
Diagrams showing how a contro protocol can change the dissipative force on the spread variables to be 'outward' (lett figure) instead of "inward' (right figure),
thus considerably altering the amount of absorbed energy, and consequenty the dyamics of the full quantum system.

states will transform into a smaller one is far more complex than those which when measured. For Dr Pattantome means that our current approaches to explaining the behaviours of nonlinear oscillators should be updated, to account for the characteristics of the measurement apparatus itself. To tackle this challenge, Dr Pattanayak and his collaborators, who include physicists at two different institutions Australia, have taken a new approach to describing weak measurements of quantum systems.

REFORMULATING A THEORY their latest research, these physicists have presented a reformulation measurement by con for weak particular setting on the lasers used to observe quantum systems. Named the 'phase angle', this setting describes a difference between the laser light emerging from a system, and a beam which has yet to pass through. For two beams with the same wavelength, the phase angle describes the distance between the points of highest and lowest ampltude between them. By adjusting the laser, this value can be changed manually.

Subsequently, the nonlinear oscillator's dynamics can be described through The first of these, nifferent values: values, describe the average of all
Until recently, quantum measurements were associated with a 'projective collapse" of the range of potential values to one specific sharp result.

possible outcomes of a measurement "Recently, we have found an elegant of the oscillating system; or, the position reformulation of the theory focusing in which an element of the system on the effects of changing the phase will be most likely to be found in at a angle setting on a laser," Dr Pattanayak given time. Secondly, the ranges in explains. "Our formulation separates positions over which these elements the quantum oscillator dynamics could possibly vary around their into those for the expectation value expectation values are named their spread variables."

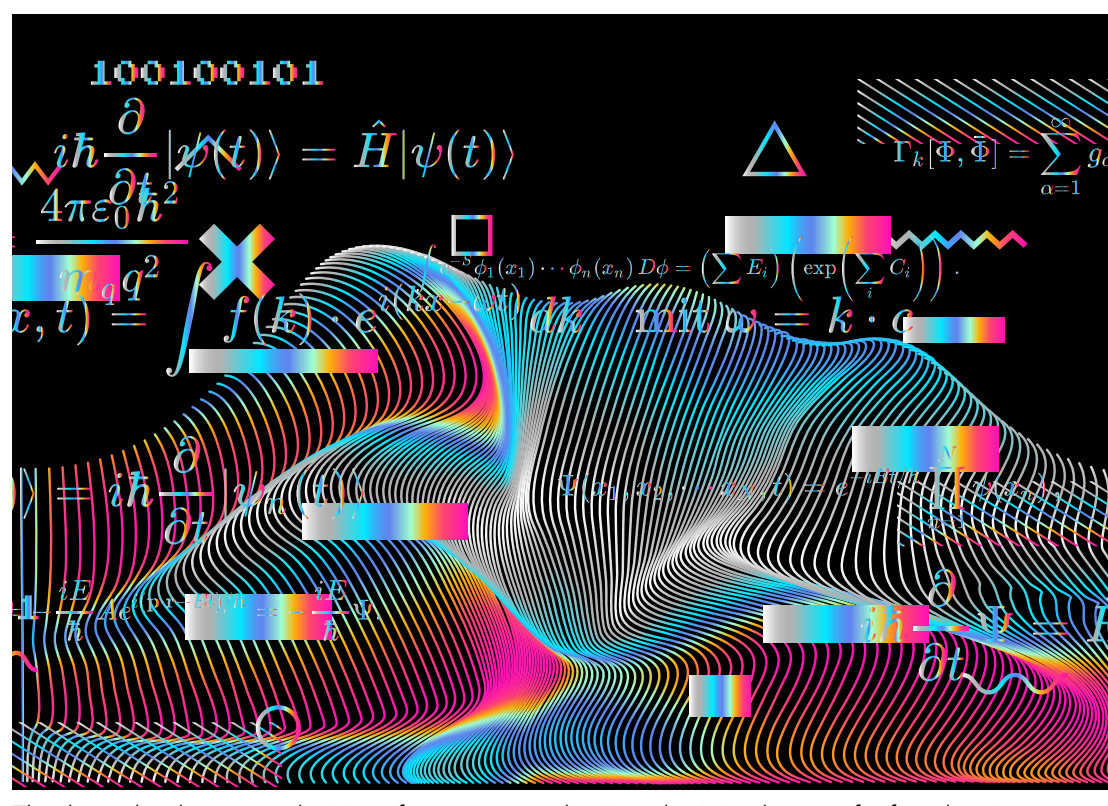

thanks to the deep complexitiles of quantum mechanics, physicicists have so far faced serious 


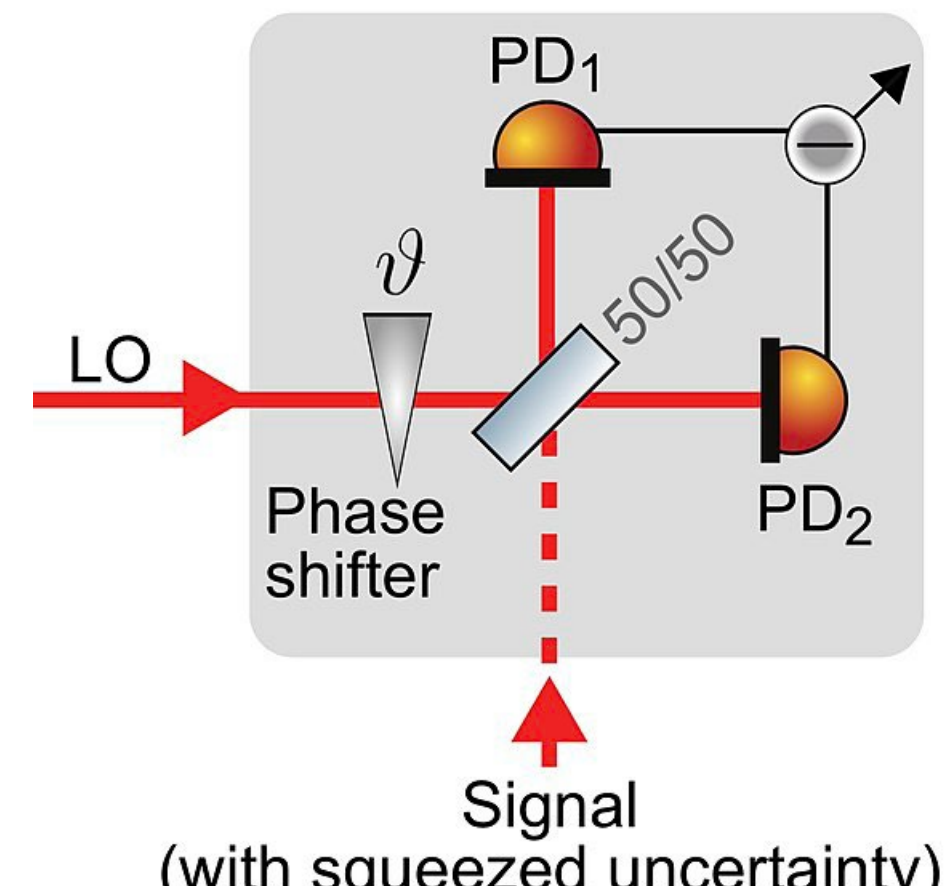

(with squeezed uncertainty)

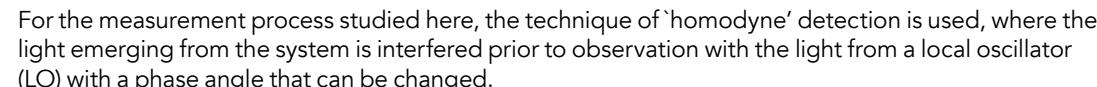

With a thorough description of how the laser affects these dynamics, other effects relating to measurements, along environment can be considered to be general fluctuations, which don't have an otherwise meaningful effect on the system's dynamics.

UNDERSTANDING ENERGY FLOWS Through this more sophisticated theory, Dr Pattanayak and his colleagues have now made new discoveries about the ways in which quaillators are affected by the energy passing through them. At particular laser phase angles, they phase angles, they
show that spread

chaotic to regular behaviour and vice versa as a function of the phase angle, depending on other system parameters This outcome is hugely significant, as it suggests for the first time that experimentalists could seize some degree of control over the dynamics of them through weak measurement, simply by adjusting the phase angle. In addition, it can be better described using classica

stems. On a qualitative level, however, eir description goes far deeper.

WIDER IMPLICATIONS FOR QUANTUM MECHANICS

Ultimately, the team's reformulated theory appears to be linked to one of the most mysterious consequences of quantum mechanics: nonlocality. Also termed 'action at a distance', this principle is in direct conflict with the currently undisputed theory of general relativity, first laid out by Einstein over a century ago. Where relativity demands that every event whic occurs in the universe must have been caused by an event in its past, limited by the finite speed of light, the researchers ppear to break this rule.

As Dr Pattanayak concludes, "our results imply that quite apart from the remarkable fect of changing the behaviour of asysem, by interacting with a signal seemingly affecting behaviour backwards in time - there could be genuinely useful consequences of enhancing the energy the spread variables." Through their future research, the physicists will continue to explore the experimental implications of their results, and the profound questions raised by systems whose dynamics are changed by future laser beams, which haven't hit them yet.

In particular, Dr Pattanayak and his it can be better described using classical adjusting phase angles when measuring
Bose-Einstein

We have found an elegant condensates-exotic reformulation of the theory focusing on the effects of changing the phase angle setting on a laser. $\quad \begin{array}{ll}\text { a large fraction of } \\ \text { these bosons can }\end{array}$ angle setting on a laser. $\quad \begin{array}{ll}\text { a large fraction of } \\ \text { these bosons can }\end{array}$ occupy the same quantum state, their quantum behaviours can be observed on macroscopic scales, allowing for a
wide range of exciting experimental wide range of exciting experimental findings promise to significantly improve the experimental opportunities for weak measurements of quantum nonlinear oscillators. For the first time, their theory suggests that researchers could gain some alters how energy flows through the to go 'outwards' to higher energy. For with the conditions of the surrounding chaotic quantum systems as they observe it implies that even if a quantum system colleagues hope to explore the effects of opportunities. For now, the team's

\section{Behind the Research} Professor Arjendu Pattanayak

W: https://apps.carleton.edu/profiles/arjendu/
Research Objectives

Arjendu Pattanayak's research interests include open complex systems.

\section{Detail}

\section{Bio}

Arjendu Pattanayak obtained his BSc from St Stephen's and PhD at the University of Texas (Austin) in 1994. Following a 3-year Post-Doctoral Fellowship at the University of Toronto he was a visiting faculty member Rice University. He then joined Carleton College Minneso in 2001 where he became Professor in the department of Physics and Astronomy in 2011

Funding

ward Hughes Medical Institute

Collaborators

Y. Shi, Dept Physics and Astronomy, Carleton College

Northfiel

S. Greenfield, Centre for Quantum Computation and Communication Technology (Australian Research Council); Centre for Quantum Dynamics, Griffith University J. K. Eastman, Centre for Quantum Computation and The Departion Technology (Australian Research Council);

Carvalho, Centre for Quantum Dynamics, Griffith University

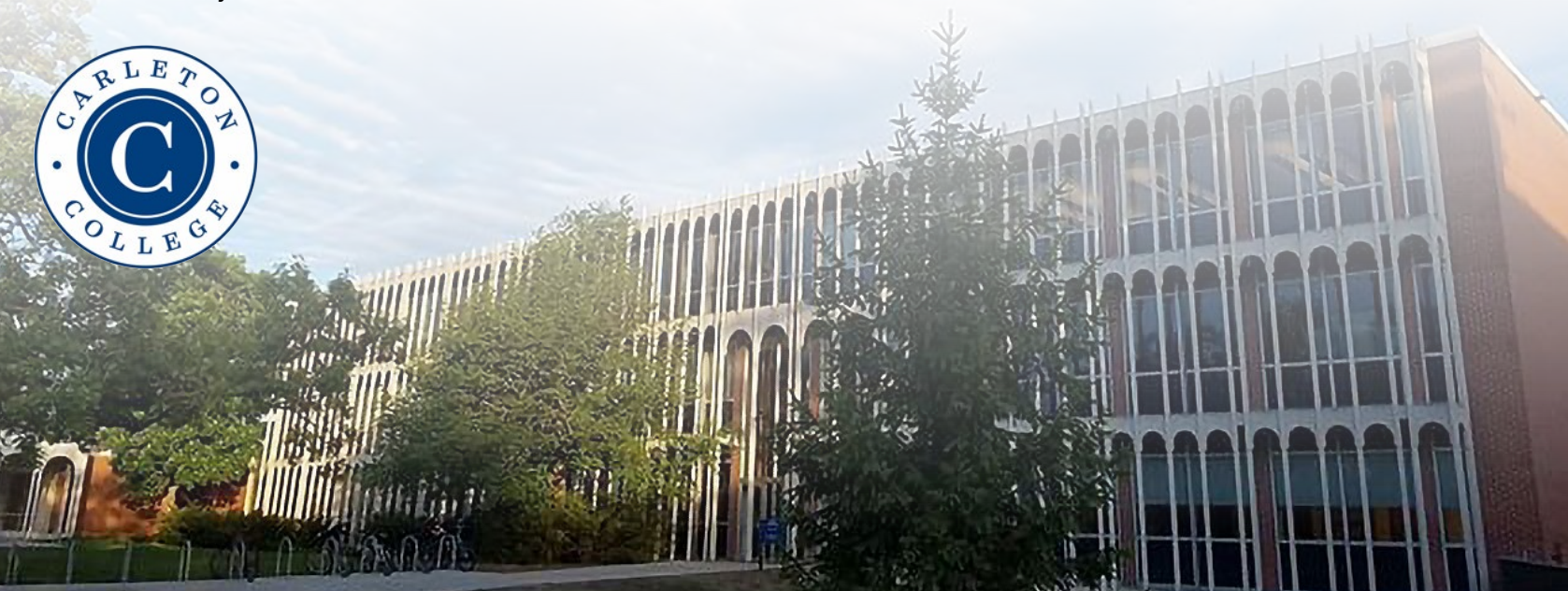

References

Shi Y, Greenfield S, Eastman JK, Carvalho ARR, Pattanayak AK. (2019). The effects of amplification of fluctuation chaotic systems: semiclassical analysis. In: Proceedings of the 5 th International Confers. Nonlinear Dynamics, p. 72-83. Springer, Cham.

Pokharel B, Misplon MZR, Lynn W, Duggins P, Hallman K, Anderson D, Kapulkin A, Pattanayak AK. (2018). Chaos and dynamical complexty in the quantum to classical 20507-w

Eastman JK, Hope JJ, Carvalho AR. (2017). Tuning quantum measurements to control chaos. Sci. Rep, 7:44684. https://arxiv.org/abs/1604.03494

\section{Personal Response}

To what extent do you think your research could impact II I hope the results help people access unusual quantum

so hope that we learn something about measur II energy scale by quantum measurement choice on quantum hitherto problematic' things like the environment and the nonlinear quantum systems, entropy, information and understand how experimental protoco the spread variables 'inward' can be changed to get the spread variables example, the system could alter from system. That is, energy dissipation to energy absorbed by spread quantum properties.

Ultimately, the quantitative des provided by the team's theory aleady and quantum effects - though further work will be required before it $c$ n and their unusual behaviour. apperne bevalid for systens which are 